\title{
Guiding students' online complex learning-task behavior through representational scripting
}

Citation for published version (APA):

Kirschner, P. A., Slof, B., Erkens, G., Jaspers, J., \& Janssen, J. (2010). Guiding students' online complex learning-task behavior through representational scripting. Computers in Human Behavior, 26(5), 927-939. https://doi.org/10.1016/j.chb.2010.02.007

\section{DOI:}

10.1016/j.chb.2010.02.007

\section{Document status and date:}

Published: 01/09/2010

\section{Document Version:}

Peer reviewed version

\section{Please check the document version of this publication:}

- A submitted manuscript is the version of the article upon submission and before peer-review. There can be important differences between the submitted version and the official published version of record. People interested in the research are advised to contact the author for the final version of the publication, or visit the DOI to the publisher's website.

- The final author version and the galley proof are versions of the publication after peer review.

- The final published version features the final layout of the paper including the volume, issue and page numbers.

Link to publication

\section{General rights}

Copyright and moral rights for the publications made accessible in the public portal are retained by the authors and/or other copyright owners and it is a condition of accessing publications that users recognise and abide by the legal requirements associated with these rights.

- Users may download and print one copy of any publication from the public portal for the purpose of private study or research.

- You may not further distribute the material or use it for any profit-making activity or commercial gain

- You may freely distribute the URL identifying the publication in the public portal.

If the publication is distributed under the terms of Article 25fa of the Dutch Copyright Act, indicated by the "Taverne" license above, please follow below link for the End User Agreement:

https://www.ou.nl/taverne-agreement

Take down policy

If you believe that this document breaches copyright please contact us at:

pure-support@ou.nl

providing details and we will investigate your claim.

Downloaded from https://research.ou.nl/ on date: 26 Apr. 2023 
Guiding Students' Online Complex Learning-task Behavior through Representational Scripting

\author{
B. Slof ${ }^{1}$, G. Erkens ${ }^{1}$, P. A. Kirschner ${ }^{2}$, J. G. M. Jaspers ${ }^{1} \&$ J. Janssen ${ }^{1}$ \\ 1Utrecht University, P.O. Box 80.140, 3508 TC Utrecht, the Netherlands \\ 2Open Universiteit Nederland, P.O. Box 2960, 6401 DL Heerlen, the Netherlands
}

Correspondence address first author:

B. Slof

Utrecht University

Research Centre Learning in Interaction

PO Box 80.140, 3508 TC Utrecht, the Netherlands

Tel: +31302533445 |Fax: +31302532352|E-mail: B.Slof@uu.nl 


\begin{abstract}
This study investigated the effects of representational scripting on students' collaborative performance of a complex business economics problem. The scripting structured the learningtask into three part-tasks, namely (1) determining core concepts and relating them to the problem, (2) proposing multiple solutions to the problem, and (3) coming to a final solution to the problem. Each provided representation (i.e., conceptual, causal, or simulation) was suited for carrying out a specific part-task. It was hypothesized that providing part-task congruent support would guide student interaction towards better learning-task performance. Groups in four experimental conditions had to carry out the part-tasks in a predefined order, but differed in the representation they received. In three mismatch conditions, groups only received one of the representations and were, thus, only supported in carrying out one of the part-tasks. In the match condition, groups received all three representations in the specified order (i.e., representational scripting). The results indicate that groups in the match condition had more elaborated discussions about the content of the knowledge domain (i.e., concepts, solutions and relations) and were better able to share and to negotiate about their knowledge. As a consequence, these groups performed better on the learning-task. However, these differences were not obtained for groups receiving only a causal representation of the domain.
\end{abstract}

Keywords: External representations, Complex learning-tasks, Computer Supported Collaborative Learning, Representational scripting, Student interaction 
Collaboratively performing complex learning-tasks such as solving complex problems is often regarded as an effective pedagogical method beneficial for both group and individual learning (Hmelo-Silver, Duncan, \& Chinn, 2007). The premise underlying this approach is that through a dynamic process of eliciting one's own knowledge, discussing this with peers, and establishing and refining the group's shared understanding of the knowledge domain, learners acquire new knowledge and skills and process them more deeply (Erkens, Jaspers, Prangsma, \& Kanselaar, 2005; Mercer, Littleton, \& Wegerif, 2004). Unfortunately, simply putting learners in a group and having them work together on a problem or a project is not always beneficial for learning (Kreijns, Kirschner, \& Jochems, 2003; P.A. Kirschner, Beers, Boshuizen, \& Gijselaers, 2008). To address this inconsistency, educators and instructional designers need to design learning situations that aim at evoking a specific kind of interaction enabling groups of learners to properly carry out their complex learning-task (Kester, Kirschner, \& Corbalan, 2007; Weinberger, Ertl, Fischer, \& Mandl, 2005).

Research on Computer Supported Collaborative Learning (CSCL) has shown that proper use of representational tools or negotiation scripts can beneficially affect interaction by stimulating learners to externalize and discuss knowledge and ideas (Fischer, Bruhn, Gräsel, \& Mand1, 2002; P.A. Kirschner, et al., 2008; Suthers, 2006). However, whereas these studies show promising results, other research questions if and how representational tools can best guide interaction when collaboratively carrying out a complex learning-task (Bera \& Liu, 2006; Elen \& Clarebout, 2007; Marjanovic, 2007; Van Drie, van Boxtel, Jaspers, \& Kanselaar, 2005). The sole presence or availability of such a tool does not in itself affect learning. The nature of the interaction depends on the interrelationship between the types of interaction that the tool is intended to support, the way the chosen tool is used by the learners, and the characteristics of the users themselves. Important here is whether the design of the tool is in line with the learners' capabilities and their intentions. That is, learners have to possess the required prior knowledge and/or skills to use the tool properly and need to experience the tool as being beneficial for carrying out their learning-task. Furthermore, the tool has to make clear to its users what they can and should do with it. Only then can learners use their prior knowledge and skills to make proper use of the tool and reach the intended learning goals (P.A. Kirschner, Martens, \& Strijbos, 2004; Veldhuis-Diermanse, 2002). Also important is the fact that complex learning-tasks are usually composed of fundamentally different part-tasks, each of which requires a different perspective on the knowledge domain (Van Bruggen, Boshuizen, \& Kirschner, 2003). The part-tasks were: (1) determining core concepts and relating them to the problem (i.e., problem orientation), (2) proposing multiple solutions to the problem (i.e., problem solution), and (3) determining suitability of the solutions and coming to a final solution to the problem (i.e., solution evaluation). Carrying out these different cognitive part-task demands should be supported by different tools. The guidance that a representational tool gives to learners carrying out a complex learning-task, therefore, needs to match the demands of the different part-tasks; otherwise this will lead to communication problems and decreased learning-task performance (Slof, Erkens, Kirschner, \& Jaspers, in press; Van Bruggen, Boshuizen, \& Kirschner, 2003).

Recently, scripting has been advanced (Dillenbourg, 2002; Weinberger, et al., 2005) as a way to ensure the alignment between tool, tool use and learning goals in collaborative learning. According to Dillenbourg a script is "a set of instructions regarding to how the group members should interact, how they should collaborate and how they should solve the problem" (p. 64). Scripting complex learning-task performance with representation tools sequences and makes the different part-task demands explicit so that they can be provided 
with congruent content-related guidance by the representational tools. By doing so, a kind of interaction beneficial for the complex learning-task performance can be evoked.

The goal of the study presented in this article is to determine whether integrating the use of scripting with the availability of representational tools leads to better use of those tools, and specifically whether this affects the interaction between group members and their complex learning-task performance in CSCL. In this article, the focus will be on guiding student interaction when collaboratively solving a complex business economics problem. One should, therefore, take into account that the congruency of the content-related guidance was tailored to the part-task demands of this problem. The premise behind the design of the representational scripting and its use, however, can be generalized to all situations where a complex learning-task has a part-task structure.

Collaboratively Solving a Complex Problem

Solving a complex problem is frequently regarded as a sequenced phased process (i.e., problem orientation phase, problem solution phase, solution evaluation phase) in which each phase has its own specific purpose and where each phase requires a specific kind of interaction (Ploetzner, Fehse, Kneser, \& Spada, 1999; Van Bruggen, et al., 2003). In the problem orientation phase, students orient themselves to the problem by constructing a cognitive bridge between their initial mental model of the knowledge domain and the mental model that needs to be created (Chi, 1997; Jonassen, 2003). This phase involves carrying out a part-task which focuses on constructing a global qualitative problem representation. This representation makes students aware of the (1) problem itself, (2) important concepts in the knowledge domain, and (3) constraints and criteria for solution of the problem and evaluation of the solution (e.g., concept A should affect concept B and this will help achieve the goal). In the problem solution phase, students must find one or more possible problem solutions. This part-task is more structured than the one in the previous phase and focuses on combining concepts in the knowledge domain into qualitative principles and making the causal relationships between the problem to be solved and the proposed solutions explicit (e.g., if concept A is increased, then concept B decreases). Here students might create a number of possible solutions and then reason about the advantages and disadvantages of each. During the third and final phase, the solution evaluation phase, students evaluate the solutions so as to choose the best one. Students need to relate the solutions with their consequences in order to determine suitability, enabling them to reach a final and suitable problem solution. This part-task focuses on evaluating the proposed solutions (e.g., making calculations) and gaining insight into their quantitative or qualitative effects and criteria (e.g., increasing concept A doubles concept B, increasing it to an unrealistic level).

Successfully solving complex problems entails students actively engaging in a process of making sense of the knowledge domain in question by articulating and discussing multiple perspectives on the problem and the problem-solving strategy (Hmelo-Silver, et al., 2007; Jonassen, 2003; Ploetzner, et al., 1999). Properly carrying out a collaborative problem-solving task requires that students interact in two different dialogical spaces (Barron, 2003; Janssen, 2008), namely the content space (i.e., carrying out part-task related activities) and the relational space (i.e., carrying out communicative activities).

Content space

In the content space, students are required to carry out part-task related activities that enable them to properly discuss the content of the knowledge domain in question. Student interaction in the content space should be aimed at acquiring a proper understanding of the knowledge domain. This requires students to carry out cognitive activities such as (1) discussing the goal of the problem-solving task/part-tasks, (2) discussing and choosing concepts, principles, and 
procedures in the knowledge domain, and (3) formulating and revising their answers and decisions (Janssen, 2008; Jonassen, 2003). Furthermore, students need to employ a proper problem-solving strategy and reflect on its suitability through carrying out meta-cognitive activities (Lazonder \& Rouet, 2008; Narciss, Proske, \& Koerndle, 2007). This means that students must discuss (1) how they should approach the problem (i.e., plan), (2) whether they have finished the part-tasks on time (i.e., monitor), and (3) the suitability of their approach (i.e., evaluate). Carrying out these cognitive and meta-cognitive activities should enable students to acquire multiple perspectives on the problem and their problem-solving strategy. However, where expert problem-solvers experience no difficulties in carrying out these kinds of activities, students (i.e., non-experts) do. When solving problems, students rely primarily on surface features such as using objects referred to in the problem instead of the underlying principles of the knowledge domain (Corbalan, Kester, \& Van Merriënboer, 2009), and employ weak problem-solving strategies such as working via a means-ends strategy towards a solution (Simon, Langley, \& Bradshaw, 1981). An important reason for this is that students lack a well developed understanding of the knowledge domain and as a consequence have problems creating and combining meaningful problem representations. This hinders students in effectively and efficiently coping with their problem-solving task because the ease with which a problem can be solved often depends on the quality of the available problem representations (Ploetzner, et al., 1999; Seufert, 2003). Different problem representations initiate different kinds of operators which act to produce new information supporting problem solvers in reaching a solution to the problem (Chi, 1997; Jonassen, 2003). To this end, it would be beneficial if suitable representations were provided and combined in a part-task appropriate manner (Ainsworth, 2006; Frederiksen \& White, 2002; Van Merriënboer, Kester, \& Paas, 2006).

Relational space

In the relational space, students carry out communicative activities enabling them to have meaningful discussions in the content space (F. Kirschner, Paas, \& Kirschner, 2009; Kreijns, et al., 2003). Such discussions are difficult, if not impossible, when students are not aware of each others' knowledge, ideas, and activities and do not discuss them with their peers.

Therefore, student interaction in the relational space is aimed at establishing and maintaining a shared understanding of the content space; a common frame of reference where conflicting points of view can be detected and negotiated (Barron, 2003; Erkens, et al., 2005; P.A.

Kirschner, et al., 2008). That is, students have to carry out communicative activities such as making their own knowledge and ideas explicit to other group members, focusing, checking and argumentation. When made explicit, students must try to maintain a shared topic of discourse and to repair a common focus if they notice a focus divergence. Simultaneously discussing different discourse topics makes it difficult to relate and understand the relevance of individual utterances, hindering establishing and maintaining a shared understanding of the content space. Students coordinate their topic of discourse by focusing (Barron; Erkens, et al.; Van Drie, et al., 2005). Also, not all concepts, principles, and procedures are relevant for carrying out a part-task, thus, students have to guard the coherence and consistency of their shared understanding of the content space. By checking, students ground their communication in a common understanding which was found to be one of the major communicative activities during collaborative problem-solving and related to the quality of the problem solving process (Van der Linden, Erkens, Schmidt, \& Renshaw, 2000). Furthermore, students must come to agreement with respect to relevant concepts principles and procedures. By using argumentation they will try to change their partners viewpoint to arrive at the best way to carry out a part-task or at a definition of concepts acceptable for all. In this argumentation process they try to convince the other(s) by elaborating on their point of view, giving 
explanations, justifications and accounts (Andriessen, Baker, \& Suthers, 2003; Erkens, et al.; P.A. Kirschner, et al.). Only when students carry out such communicative activities their interaction can be sufficiently coordinated and multiple perspectives on the problem and the problem-solving strategy can arise.

\section{Guiding Student Interaction through Representational Scripting}

Integrating scripting with the availability of representational tools (i.e., representational scripting) is intended to structure the problem-solving process making it more efficient and effective. Scripting shapes the use of the representational tools and, therefore, also the epistemic and social processes of collaboration (Slof, et al., in press; Weinberger, et al., 2005) by sequencing and making the different part-task demands explicit so that they can be provided with part-ask congruent support by the representational tools. Each representation tool provides a different domain-specific content scheme (i.e., problem representation) representing different perspectives on the problem. Visualizing the knowledge domain by providing multiple external representations (ERs) influences student interaction through their representational guidance (Suthers, 2006; Van Drie, et al., 2005). Due to its ontology (i.e., objects, relations, and rules for combining them), every ER offers a restricted view of the knowledge domain, guiding student interaction in a specific manner. Matching the representational guidance of the ERs (see Table 1) with the student interaction required to carry out a part-task evokes proper part-task related and communicative activities, leading to more successful group learning-task-performance. To effectively do this, one must avoid or neutralize the difficulties students encounter when combining multiple ERs, namely problems translating from and coordinating between different kinds of ERs (Ainsworth, 2006), and incongruence between ER and part-task related activities (Vekiri, 2002). This means that the representational guidance of the ER provided in a specific representational tool must be congruent (i.e., ontologically matched) with the part-task demands and activities of a specific problem phase (Schnotz \& Kürschner, 2008; Van Bruggen, et al., 2003).

$* * * *$ INSERT TABLE 1 ABOUT HERE****

Design and Research Questions

This study focuses on how the use of representational scripting affects both student interaction and group learning-task performance in a CSCL-environment. To this end, four experimental conditions were defined. In triads, students in all conditions had to collaboratively solve a case-based problem in business-economics which was divided into three problem phases, each coupled with different ERs. To study the effects of the representational scripting, the ERs were either matched or mismatched to the different problem phases (see Table 2).

\section{****INSERT TABLE 2 ABOUT HERE****}

The scripting structured the problem-solving process in three phases, but only one of the three ERs was made available to the students when carrying out a specific part-task. In three mismatch conditions, student groups only received one of the ERs (i.e., conceptual ER causal ER, simulation ER) and had to use this ER for carrying out all three part-tasks. The provided ER ontologically matched only one of the three part-tasks and there was a mismatch for the other two. In the fourth condition, student groups received all three ERs in a phased order, receiving the ER most suited to each part-task. Here, thus, there was a match between all three ERs and all three part-tasks. Student groups in this condition received the complete array of representations. Due to the presumed match between ERs and part-tasks, student understanding, part-task related activities and communicative activities were expected to increase, allowing the students to reach better problem solutions. It was, therefore, hypothesized that students in the match condition: 
(H1) interact more according the requirements of both dialogue spaces, they are expected to:

a) carry out more cognitive and meta-cognitive activities,

b) have more elaborated discussion of the content of the knowledge domain,

and

c) carry out more communicative activities to coordinate their interaction in

the content space.

(H2) arrive at better solutions to the problem.

Method

\section{Participants}

Participants were students from six business-economics classes in three secondary education schools in the Netherlands. The total sample consisted of 96 students (59 male, 37 female). The mean age of the students was 16.67 years $(S D=0.77$, Min $=15$, Max $=18)$. Students were, within classes, randomly assigned to a total of 32 triads, which were equally divided between the four experimental conditions.

\section{Materials}

\section{Collaborative learning environment}

Students worked in a CSCL-environment called Virtual Collaborative Research Institute (VCRI, see Figure 1), a groupware application for supporting the collaborative performance of problem-solving tasks and research projects (see Erkens, et al., 2005). For this study, five tools that are part of the VCRI were augmented with representational scripting. All tools, except the Notes tool, were shared among group members.

****INSERT FIGURE 1 ABOUT HERE****

The Chat tool enables synchronous communication and supports students in externalizing and discussing their knowledge and ideas. The chat history is automatically stored and can be reread by the students. In the Assignment menu, students can find the description of the problem-solving task/part-tasks. Besides this, additional information sources such as a definition list, formula list, and clues for solving the problem were also available here. The Co-writer is a shared text-processor where students can collaboratively formulate and revise their answers to the part-tasks. The Notes tool is an individual notepad that allows students to store information and structure their own knowledge and ideas before making them explicit. The Status bar is an awareness tool that displays which group members are logged into the system and which tool a group member is currently using. All students in all conditions had access to these tools and information sources. In other words, the different conditions were information equivalent and only differed in the way that the ERs are intended to guide the interaction and group learning-task performance. Problem-solving task

All groups worked on a case-based problem in business-economics in which they had to advise an entrepreneur about changing the business strategy to increase profits (i.e., company result). Through the use of scripting, the problem-solving process was structured into a 
problem orientation phase, a problem solution phase, and a solution evaluation phase each focusing on one of the part-tasks. To come up with a suitable advice, students had to carry out three different part-tasks, namely (1) determine the main factors that determine the company's results and relate them to the problem, (2) determine how certain interventions such as changing the business strategy affect company results, and (3) compare the effects of these interventions and formulate a final advice based on this comparison. All groups were 'forced' to carry out the part-tasks in a predefined order; they could only start with a new part-task after finishing an earlier part-task. When group members agreed that a part-task was completed, they had to 'close' that phase in the assignment menu. This 'opened' a new phase, which had two consequences for all groups, namely they (1) received a new part-task, and (2) had to enter their new answers in a different window of the Co-writer and could not alter their prior answers though these answers remained visible. All conditions received the part-tasks in the same order (i.e., used the same script), but differed in the content-related guidance they received. Groups in the mismatch conditions used an ER that was only suited to one of the three phases/part-tasks and had to use this ER for carrying out all three part-tasks. In contrast, groups in de match condition received a different ER for each phase/part-task which was ontologically matched to the specific demands of that phase. The congruency of the contentrelated guidance of the ERs was developed in cooperation with the teachers of the participating schools and has been validated in a small scale pilot study. For a more thorough theoretical description of the congruency between ERs and part-tasks and its effects on group and individual learning, the reader is referred to Slof et al. (in press).

The problem orientation phase focused on creating a global qualitative problemrepresentation by asking students to explain what they thought the problem was and to describe what the most important factors were that influenced the problem. During this phase, students received the conceptual ER (i.e., a static content scheme; see Figure 2) which made two aspects salient, namely the core concepts needed to carry out this part-task and which core concepts are qualitatively related to which other core concepts. Students could, for example, see that 'company result' is determined by the 'total profit' and the 'efficiency result'. Such information should make it easier for them to create an overview of all relevant concepts (i.e., to broaden the problem space), supporting them in finding multiple solutions to the problem in the following phase. The conceptual ER available in this phase supports the creation of a global qualitative problem representation which can be elaborated on in the following problem phases.

\section{****INSERT FIGURE 2 ABOUT HERE****}

The problem solution phase focused on creating a causal problem representation (i.e., explicating the underlying business-economics principles) by asking students to formulate several solutions to the problem. During this phase, students received the causal ER (i.e., a static content scheme; see Figure 3), in which the causal relationships - visible through the arrows showing direction of the relationship between the concepts - were specified. The causal ER also contributed to increasing students' qualitative understanding by providing the students with possible interventions (i.e., changes in the business strategy), each of which had a different effect on the company results. This should make it easier to effectively explore the solution space and should, in turn, support students in finding multiple solutions to the problem. Students could, for example, see that a PR-campaign affects 'actual sales, which in turn affects 'total profit'. The conceptual ER used in the first phase is not expressive enough for this part-task because the relations in that ER were not specified and students did not receive information about possible interventions, forcing them to produce the advice themselves without sufficient understanding of the underlying qualitative principles of the knowledge domain.

$$
\text { ****INSERT FIGURE } 3 \text { ABOUT HERE**** }
$$


Finally, the solution evaluation phase focused on increasing the students' understanding of the knowledge domain with the aid of a quantitative problem representation. Students were asked to determine the financial consequences of their proposed solutions and to formulate a final advice for the entrepreneur by negotiating the suitability of the different solutions with each other. During this phase, students received a simulation ER (i.e., a dynamic content scheme; see Figure 4) which enabled them to simulate the financial consequences of their proposed solutions, by clicking on the arrows in the boxes. When adjusting (i.e., increasing or decreasing) the height or the quantity of a certain value, the simulation model automatically computed the values of the other concepts. This is meant to facilitate the determination and negotiation of the suitability of the different proposed solutions and reaching a final advice. Students could, for example, test how the PR-campaign affects the 'actual sales' and whether this in turn affects the 'total profit'. Only the simulation ER is capable of providing this kind of support, because the relationships between the concepts in this ER were specified as equations.

\section{****INSERT FIGURE 4 ABOUT HERE****}

\section{Procedure}

All student groups spent three, 70-minute lessons solving the problem during which each student worked on a separate computer in a computer classroom. Before the first lesson, students received an instruction about the CSCL-environment, the group composition, and the problem-solving task. The instruction made it clear that their group answer to the problem (i.e., learning-task performance) would serve as a grade affecting their GPA. Students worked on the problem in the computer classroom where all actions and answers to the part-tasks were logged. During the lessons, the teacher was on stand-by for learning-task related questions and a researcher was present for technical support.

\section{Measurement of student interaction}

To examine the effect of condition data concerning student interaction were collected by logging the chat-utterances of the group members. The content of these chat-protocols is assumed to represent what students know and consider important for carrying out their problem-solving task (Chi, 1997; Moos \& Azevedo, 2008). To properly analyze these data, researchers should use a combination of qualitative and quantitative methods (Chi; De Wever, Van Keer, Schellens, \& Valcke, 2007; Mercer, et al., 2004). This allows the qualitative (i.e., contextual) nature of student interaction to be quantified (i.e., systematically coded and compared) enabling researchers to account for differences in learning-task performance and generalize their results. Using so called 'concordancers' software (e.g., MEPA, Erkens, 2005; !Kwictex, Mercer, et al.) minimizes the work associated with coding the chat-protocols and maximizes coding allowing the content of chat-protocols to be searched for the occurrence of important words or phrases within their linguistic context to show their specific function in the dialogue.

\section{Level of analysis and segmentation}

The chat-protocols were selected and transferred from the log-files using the Multiple Episode Protocol Analysis (MEPA) program (Erkens, 2005). MEPA uses a multidimensional data structure, allowing chat-protocols to be segmented into multiple levels for analysis, here the episodic level and the event level. Measurement at the episodic level provides insight into the number and nature of the discourse topics that students discussed. An episode is regarded as a dialogue between minimally two students in which a distinct discourse topic is discussed and which ends with a confirmation by at least two students that they understood each other (Erkens, et al., 2005). For example, discussing the suitability of a problem-solving strategy 
requires the involvement of multiple students who each use more than one utterance to make their point. At the episodic level only the discourse topic itself is coded. Measurement at the event level provides insight in the discussion of the content of the knowledge domain and the communicative function of utterances. To this end, this analysis took place at a finer grain size, namely the utterance (Chi, 1997; Erkens, et al.; Mercer, et al., 2004). For example, discussing a concept such as 'costs' is particularly important when solving the problem used in this study. A problem here is that even within in a single sentence, multiple concepts or statements may be expressed and thus require multiple codes (Erkens \& Janssen, 2008; Strijbos, Martens, Prins, \& Jochems, 2006). With a MEPA-filter which makes use of 300 structured 'if-then' decision rules, the utterances were automatically segmented into smaller, still meaningful, subunits. Punctuation marks (e.g., full stop, exclamation mark, question mark, comma) and connecting phrases (e.g., 'and if', or 'but if') were used to segment the utterances. At the event level, all utterances relevant for the analysis are coded separately.

Content space: Coding schemes and reliability-measures

In the content space, students carry out part-task related activities that enable them to properly discuss the content of the knowledge domain in question. Two coding schemes were applied to gain insight in the student interaction. Measurement at the episodic level provided insight in the cognitive, meta-cognitive and off-task activities students carried out during collaborative problem solving. Student interaction was segmented into different discourse topics which coding led to the measurement of the part-task related and off-task activities (see Table 3). The discourse topics were hand-coded and Cohen's kappa was computed for three chat-protocols which were coded independently (total of 3532 lines) by two coders. An overall Cohen's Kappa of .70 was found, an intermediate to good result (Cicchetti, Lee, Fontana, \& Dowds, 1978).

****INSERT TABLE 3 ABOUT HERE****

Measurement at the event level provided insight into student interaction with respect to discussion of the content of the knowledge domain. A learning-task analysis based on the work of Gagné, Wagner and Briggs (1992) was conducted and resulted in 17 businesseconomics concepts and 9 possible interventions (see, for example, Figure 3). To make coding and analyses more manageable, concepts and solutions were categorized into five and three subcategories respectively. Furthermore, three possible ways of interrelating the concepts and solutions were included, resulting in the coding scheme in Table 4. $* * * *$ INSERT TABLE 4 ABOUT HERE****

The chat-protocols were automatically searched for the occurrence of characteristic words which led to the identification and coding of the dependent variables (Erkens \& Janssen, 2008; Mercer, et al., 2004). This was done automatically with a MEPA-filter which makes use of 900 structured 'if-then' decision rules containing different explicit references to a concept, solution or relation (e.g., name, synonyms, etc.) which were coded as representing that concept, solution or relation. Through a process of testing and adapting the filter, overall Cohen's Kappa for concepts, solutions and relations ranging from .70.to .86, were reached compared to hand-coding four chat-protocols (total of 4198 lines). These finding indicate that this automatic coding procedure is reliable.

Relational space: coding scheme and reliability-measure

In the relational space, students carry out communicative activities to properly manage their interaction in the content space. Each utterance was coded with respect to the type of dialogue act used (see Table 5). A dialogue act is regarded as a communicative action which is elicited for a specific purpose representing a specific function in the dialogue (Erkens, et al., 2005; Erkens \& Janssen, 2008). The dialogue acts were: 
- $\quad$ argumentatives; indicating a line of reasoning,

- $\quad$ responsives; indicating responses to questions or proposals,

- $\quad$ informatives; indicating a transfer of information, often statements or evaluations,

- $\quad$ elicitatives; indicating questions or proposals requiring an answer,

- $\quad$ imperatives; indicating commands to take action or to draw attention.

****INSERT TABLE 5 ABOUT HERE****

The coding of the dialogue acts took place at the event level and was based on the occurrence of characteristic words or phrases (i.e., discourse markers, see Schiffrin, 1987) which indicates the communicative function of an utterance. The chat-protocols were searched for the occurrence of these discourse markers which led to the identification and coding of the dependent variables (Erkens \& Janssen, 2008; Mercer, et al., 2004). This was automatically done with a MEPA-filter using 1,250 structured 'if-then' decision rules that uses pattern matching to find typical words or phrases. When compared to hand-coding, an overall agreement of 79\% was reached and a Cohen's Kappa of .75 was found (Erkens \& Janssen, 2008). This finding indicates that the automatic coding procedure is reliable. After coding, score-frequencies for each dialogue act were computed and combined resulting in the measurement of the variables focusing, checking and argumentation. Measurement of learning-task performance; coding scheme and reliability

To examine the effect of the condition on group learning-task performance, an assessment form was developed. Table 6 provides a description of the aspects on which the answers were evaluated, the number of items, and their internal consistency scores (Cronbach's alpha). The problem-solving task consisted of three part-tasks in which the groups each had to answer three questions. All nine answers were evaluated based on their 'suitability', 'elaboration', 'justification', and 'correctness', resulting in 36 items ( 9 answers $\times 4$ criteria). Also evaluated was whether groups used answers from a subsequent phase and altered their way of reasoning when they had to answer the questions asked in a following phase (i.e., 'continuity'). There were two phase transitions (i.e., transition from problem orientation to problem solution and transition from problem solution to solution evaluation) and, therefore, two items (2 items). Finally, the 'quality of the final advice' was evaluated by three items; number of concepts incorporated in the advice, financial consequence of the advice, and whether the final answer was in line with the guidelines provided in the original task description. This resulted in a total of 41 items which all could be coded as ' 0 ' (wrong), ' 1 ' (adequate) or '2' (good); the higher the code, the higher the quality of the answer. Groups could, thus, achieve a maximum score of 82 points for their learning-task performance (41 items $\times 2$ points) and a minimum of 0 points. The internal consistency score for the whole complex learning-task performance was .89 and for most subscales, internal consistency scores of .60 or above were obtained.

$* * * *$ INSERT TABLE 6 ABOUT HERE****

\section{Data analysis}

When conducting studies on CSCL, group members mutually influence each other (i.e., behave more or less in the same way) leading to non-independence of measurement (De Wever, et al., 2007; Kenny, Kashy, \& Cook, 2006). This is problematic because many 
statistical techniques (e.g., $t$-test, ANOVA) assume score-independence and such a violation compromises the interpretation of the output of their analyses (e.g., $t$-value, standard error, $p$ value, see Cress, 2008; Janssen, 2008; Kenny, et al.). This non-independence requires a statistical technique that addresses this. Multilevel analysis (MLA) is an approach suited to "appropriately grasp and disentangle the effects and dependencies on the individual level, the group level, and sometimes the classroom level" (Strijbos \& Fischer, 2007, p. 391). The nonindependence was determined here by computing the intraclass correlation coefficient and its significance (Kenny, et al.) for all dependent variables concerning student interaction. This coefficient demonstrated non-independence $(\alpha<.05)$ for all tests, justifying MLA for analyzing these data. MLA entails comparing the deviance of an empty model and a model with one or more predictor variable(s) to compute a possible decrease in deviance. The latter model is considered a better model when there is a significant decrease in deviance in comparison to the empty model (tested with a $\chi^{2}$-test). Almost all reported $\chi^{2}$-values were significant $(\alpha<.05)$ and, therefore, the estimated parameters of these predictor variables (i.e., effects of condition) were tested for significance. An one-way MANOVA was used for answering the second research question concerning the influence of representational scripting on the quality of the solutions produced by the groups.

Results

Testing the assumptions of the statistical techniques led to the detection of several outliers. That is, the obtained scores differed three or more $S D s$ from the grand mean concerning the analyses of the cognitive, meta-cognitive and off-task activities (three students) and concepts, solutions and relations, and the communicative activities (four students). The utterances of those students were, therefore, deleted from the specific MLAs. Since there were specific directions of the results expected (see hypotheses) all analyses are one-sided.

Cognitive, meta-cognitive and off-task activities

MLAs revealed that condition was not a predictor for students' meta-cognitive activities ( $\beta$ $=6.82, p=.09)$, cognitive activities $(\beta=1.04, p=.33)$, and off-task activities $(\beta=0.61$, $p=.30$ ) when comparing students in the match condition to students in the non-matched conditions. The mean scores, standard deviations and condition effects (i.e., difference between match condition and non-matched conditions) for the different kinds of discourse topics are listed in Table 7.

When analyzing the different discourse topics for the conditions separately, several condition effects were found. First, a category effect for cognitive activities was found when comparing students in the match condition to students in the simulation condition $(\beta=5.39, p=.02)$. As indicated by the ${ }^{+}$and ${ }^{-}$signs in Table 7 , students in the match condition exhibited more cognitive activities in comparison to students in the simulation condition. Students in the simulation condition discussed fewer content-related discourse topics and formulated/revised their answers to the part-task (i.e., content) less often in comparison to students in the match condition $(\beta=3.79, p=.03)$. Furthermore, students in the simulation condition discussed less whether or not they should end a part-task (i.e., logging) than students in the match condition $(\beta=1.25, p=.02)$. Finally, students in the match condition exhibited more technical-related activities than those in the non-matched conditions $(\beta=0.50, p=.05)$. When comparing the match condition to the other conditions separately, the effects were significant for the causal $(\beta=0.39, p=.02)$ and the simulation condition $(\beta=0.37, p=.02)$.

As expected, students in the match condition carried out more cognitive activities than students in the simulation condition. That is, they had more discussion about the content of the knowledge domain and formulated, revised and registered more of their answers. These differences were, however, not found for the comparison to students in the conceptual and the 
causal condition. Although expected, students in the match condition did not discuss their problem-solving strategy, its suitability and whether their part-tasks were finished on time more often than students in the non-matched conditions.

****INSERT TABLE 7 ABOUT HERE****

\section{Concepts, solutions and relations}

MLAs revealed that condition was a predictor for the number and kinds of concepts, solutions and relations that students discussed. The mean scores, standard deviations and condition effects (i.e., difference between match condition and non-matched conditions) for the discussion of the concepts, solutions and relations are listed in Table 8.

First, a marginally significant category effect for concepts was found; students in the match condition discussed more concepts in comparison to those in the non-matched conditions ( $\beta$ $=5.27, p=.08$ ). When analyzing the different concepts separately, it appeared that students in the match condition discussed a concept as 'sales' more often than students in both the conceptual $(\beta=1.97, p=.04)$ and the causal condition $(\beta=2.79, p=.01)$. Furthermore, students in the match condition discussed a concept as 'costs' more often than students in both the conceptual $(\beta=2.46, p=.08)$ and the simulation condition $(\beta=2.78, p=.05)$. It should be noted that this difference was only marginally significant for students in the conceptual condition. Second, a significant category effect for solutions was found; students in the match condition discussed more solutions in comparison to students in the non-matched conditions $(\beta=4.96, p=.05)$. When analyzing the different kinds of solutions separately, it appeared that students in the match condition had a tendency of discussing solutions aimed at (1) decreasing the company's costs more often than students in both the conceptual $(\beta=1.97$, $p=.06)$ and the causal condition $(\beta=2.12, p=.06)$, and (2) increasing the company's turnover more often than students in the simulation condition $(\beta=2.37, p=.02)$. Finally, a marginally significant category effect for relationships was found; students in the match condition discussed more and different kinds of relationships than students in the nonmatched conditions $(\beta=5.32, p=.08)$. This was caused mainly by the fact that students in the match condition discussed mathematical relationships more often than students in the nonmatched conditions $(\beta=0.72, p=.02)$. When comparing the match condition to the other conditions separately, it appeared that this was (1) the case for students in the conceptual $(\beta$ $=0.71, p=.02)$ and (2) marginally the case for students in the simulation condition $(\beta=0.73$, $p=.06)$.

As expected, students in the match condition discussed more important concepts for solving the problem than students in the non-matched conditions. Concepts as 'sales' and 'costs' are vital because they deal with how much income and expenses a company has (i.e., variables that determine the profitability of a company). Students in the match condition also discussed more solutions than students in the non-matched conditions. This yielded - especially for solutions aimed at increasing the income or decreasing the costs of a company - a result in line with discussing concepts as 'sales' and 'costs' more often. Furthermore students in the match condition also related the concepts to each other and to the solutions more often than students in the non-matched conditions. When doing this, they made use of different kinds of relationships, namely conceptual, causal and mathematical ones.

****INSERT TABLE 8 ABOUT HERE****

\section{Communicative activities}

MLAs revealed that condition was a predictor for the management of the interaction in the content space. The mean scores, standard deviations and condition effects (i.e., difference between match condition and non-matched conditions) for the communicative activities are listed in Table 9. 
A marginally significant effect for coordination was found, namely that students in the match condition had a tendency of exhibiting more communicative activities compared to students in the non-matched conditions $(\beta=17.96, p=.07)$. For the specific communicative activities, the following results were obtained. First, a marginally significant category effect for checking was found; students in the match condition devoted more attention to guarding the coherence and consistency of their shared understanding of the content space than students in the non-matched conditions $(\beta=8.80, p=.07)$. However, when comparing the match condition to the other conditions separately, no significant results were obtained. Finally, a significant category effect was found for argumentation; students in the match condition exhibited more argumentative activities than students in the non-matched conditions ( $\beta$ $=6.20, p=.05)$. When comparing the match condition to the other conditions separately, this was only marginally the case for the conceptual condition $(\beta=7.00, p=.08)$.

As expected, students in the match condition were better able to establish and maintain shared understanding of the content space and to negotiate about it than students in the non-matched conditions. This should have enabled students in the match condition to acquire multiple perspectives on the problem and the problem-solving strategy, which are both seen as beneficial to problem-solving. Although, in total, students in the match condition exhibited more communicative activities, more differences concerning specific communicative activities between conditions were expected.

$$
* * * * \text { INSERT TABLE } 9 \text { ABOUT HERE**** }
$$

\section{Learning-task performance}

An one-way MANOVA on the total score on the complex learning-task performance showed a significant difference for condition $(F(3,28)=1.72, \mathrm{p}=.03$; Wilks' Lambda $=0.33$; partial eta squared $=.31)$. Bonferroni post hoc analyses showed that groups in the match condition scored significantly higher than groups in both the conceptual $(p=.00 ; d=2.19)$ and the simulation condition $(p=.04 ; d=1.26)$. Table 10 shows the mean scores and standard deviations of the scores on the group learning-task performance.

When the results for the dependent variables were considered separately, using one-way ANOVAs with Bonferroni post hoc analyses, condition effects were found for suitability $(F(3,28)=2.99, \quad p=.03)$, justification $(F(3,28)=4.23, \quad p=.01)$ and correctness $(F(3,28)=2.99, p=.03)$. The mean scores indicated that there were several significant differences between conditions. First, groups in the match condition scored significantly higher on suitability than groups in the conceptual condition $(p=.01 ; d=1.45)$ and a trend was found in comparison to the groups in the simulation condition $(p=.07 ; d=0.77)$. Second, groups in the match condition scored significantly higher on justification than groups in both the conceptual $(p=.01 ; d=1.53)$ and the simulation condition $(p=.02 ; d=1.29)$. Finally, groups in the match condition scored significantly higher on correctness than groups in the conceptual condition $(p=.03 ; d=1.83)$ and a trend was found in comparison to the groups in the simulation condition $(p=.06 ; d=1.04)$.

These result confirmed our second hypothesis, namely that groups that received an ontologically congruent ER for each phase-related part-task scored higher on the group learning-task performance.

\section{****INSERT TABLE 10 ABOUT HERE****}

Discussion

As is the case with many other researchers, the present study stresses the importance of using representational tools (Fischer, et al., 2002; Schnotz \& Kürschner, 2008) and of employing scripting (P.A. Kirschner, et al., 2008; Weinberger, et al., 2005) to guide student interaction and learning-task performance. However, instead of using them separately, this study combined the advantages of using multiple representational tools and scripting (i.e., 
representational scripting). The representational scripting structured the problem-solving process by sequencing and making the part-tasks explicit so that they could be provided with ontologically congruent content-related guidance in the representational tools. It was hypothesized that this would evoke more task-suited student interaction and, as a consequence, better group learning-task performance than not receiving it. The results of our study confirmed that the problem-solving process for these student teams was more efficient and effective. The representational scripting shaped the use of the representational tools and guided student interaction towards acquiring and applying suitable qualitative and quantitative problem representations. Those activities are often regarded as beneficial for collaborative problem-solving (Hmelo-Silver, et al., 2007; Jonassen, 2003; Ploetzner, et al., 1999). Specifically, groups in the match condition (1) had more elaborated discussions of the content of the knowledge domain, and (2) were also better able to establish and maintain their shared understanding of the knowledge domain, a prerequisite for a proper discussion of it, than students in the non-matched conditions. As a consequence, the groups receiving an ontologically congruent ER for each part-task (i.e., match condition) gave better answers to the part-tasks and came up with better final solutions to the problem than groups in the nonmatched conditions.

Although the results seem very promising, there were some contrasting findings that require further discussion. First, student interaction in the causal condition only slightly differed from that of the students in the match condition and their group learning-task performance was also very similar to what was found in the match condition. Students in both conditions received the causal ER, which showed all relevant concepts, solutions and their causal interrelationships, providing the students with multiple qualitative perspectives on the knowledge domain. It seems, therefore, important to recognize that causal reasoning is beneficial for collaborative problem-solving (Jonassen \& Ionas, 2008). However, it does not completely explain the lack of differences. Perhaps combining the causal ER with both the conceptual and the simulation ER hinders problem-solving when students experience difficulties integrating the different ERs. When students do not know how to use an ER and/or combine multiple ERs, they might choose to stick with the familiar one and make no attempt to integrate the different ERs (Ainsworth, 2006; Bodemer \& Faust, 2006). Our design and collected data enables us to study whether students used all provided ERs and how students in the match condition integrated the different ERs. This kind of process data might reveal whether and how students used the multiple concepts, solutions and qualitative and quantitative ways of interrelating them, providing more insight into the effects of the ontology of the different ER's. However, due to space limitations and time constraints, the analyses of these data were not yet incorporated in the current article. Second, students in the match condition did not discuss their problem-solving strategy, its suitability and whether their parttasks were finished on time more often than students in the non-matched conditions. Carrying out these meta-cognitive activities might not differ between because the scripting, which was the same for all conditions, structured the problem-solving process into three phases, each focusing on one of the part-tasks and thereby affecting students' problem-solving strategy (Dillenbourg, 2002; P.A. Kirschner, et al., 2008).

Implications and Future Research

This study has several implications for learning-environment design (e.g., CSCLenvironment) for supporting students in carrying out complex learning-tasks. Collaborative problem-solving is facilitated and learning-task performance is better when the different parttasks are made explicit, are properly sequenced, and each is provided with an ontologically congruent domain-specific content scheme. Using multiple ERs provides different perspectives of the knowledge domain and, when matched to the part-task demands and 
activities, the complementary function of those ERs can gradually increase students understanding, and evoke proper part-task related and communicative activities (see Ainsworth, 2006). However, when interpreting the results and the implications of this study, one has take into consideration that it was aimed at supporting students collaboratively solving a problem in the field of business-economics. That is, a specific kind of complex learning-task was used and situated in a specific knowledge domain. Although there are many other knowledge domains (e.g., physics, urban planning) and complex learning-tasks (i.e., inquiry tasks, research projects) in which different part-tasks have to be carried out, the manner in which the use of representational tools is shaped depends on the specifics of the complex learning-task and the knowledge domain. The effect of the design of representational scripting and its use does, therefore, not automatically apply to all knowledge domains and all kinds of complex learning-tasks.

Taking the contrasting findings and the limitations into mind, additional research into the effects of representational scripting should be carried out to investigate whether these results can be generalized to other knowledge domains and other types of complex learning-tasks. Future research should also focus on gaining more insight into the effects of different ontology's by studying how students co-construct their own ERs. Such an approach entails that students co-construct and adjust their own representations of the knowledge domain and are, therefore, more occupied with interrelating the different concepts and solutions than when the ERs are provided. Those studies probably provide more insight in the manner in which students actually use the different concepts, solutions and ways of interrelating them. Furthermore, co-constructing and adjusting their own ERs might also provide more support for developing a better developed understanding of the knowledge domain leading to more individual learning gains. Finally, research on CSCL should measure both student interaction and learning-task performance because this can provide a deeper understanding of the effects of the provided guidance (i.e., tools and/or strategies). This understanding should enable researchers to determine how (1) the design of the guidance affects student interaction, and (2) this kind of guidance supports students in carrying out complex learning-tasks and learning from them.

\section{References}

Ainsworth, S. (2006). DeFT: A conceptual framework for considering learning with multiple representations. Learning and Instruction, 16, 183-198.

Andriessen, J., Baker, M., \& Suthers, D. D. (2003). Argumentation, computer support, and the educational context of confronting cognitions. In J. Andriessen, M. Baker, \& D. D. Suthers, (Eds.), Cognitions: Arguing to learn (pp. 1-25). Dordrecht, The Netherlands: Kluwer Academic Press.

Barron, B. (2003). When smart groups fail. Journal of the Learning Sciences, 12, 307-359.

Bera, S., \& Liu, M. (2006). Cognitive tools, individual differences, and group processing as mediating factors in a hypermedia environment. Computers in Human Behavior, 22, 295-319.

Bodemer, D., \& Faust, U. (2006). External and mental referencing of multiple representations. Computers in Human Behavior, 22, 27-42. 
Chi, M. T. H. (1997). Quantifying qualitative analyses of verbal data: A practical guide. Journal of the Learning Sciences, 6, 271-315.

Cicchetti, D. V., Lee, C., Fontana, A. F., \& Dowds, B. N. (1978). A computer program for assessing specific category rater agreement for qualitative data. Educational and Psychological Measurement, 38, 805-813.

Corbalan, G., Kester, L., \& Van Merriënboer, J. J. G. (2009). Selecting learning tasks: Effects of adaptation and shared control on learning efficiency and task involvement. Contemporary Educational Psychology, 33, 733-756.

Cress, U. (2008). The need for considering multilevel analysis in CSCL research - An appeal for the use of more advanced statistical methods. International Journal of ComputerSupported Collaborative Learning, 3, 69-84.

De Wever, B., Van Keer, H., Schellens, T., \& Valcke, M. (2007). Applying multilevel modelling to content analysis data: Methodological issues in the study of role assignment in asynchronous discussion groups. Learning and Instruction, 17, 436447.

Dillenbourg, P. (2002). Over-scripting CSCL: The risks of blending collaborative learning with instructional design. In P. A. Kirschner (Ed.), Three worlds of CSCL: Can we support CSCL? (pp. 61-91). Heerlen, The Netherlands: Open Universiteit Nederland.

Elen, J., \& Clarebout, G. (2007). Supporting learning: Increasing complexity? Computers in Human Behavior, 23, 1162-1166.

Erkens, G. (2005). Multiple Episode Protocol Analysis (MEPA). Version 4.10. Utrecht University, The Netherlands.

Erkens, G., \& Janssen, J. (2008). Automatic coding of online collaboration protocols. International Journal of Computer-Supported Collaborative Learning, 3, 447-470. 
Erkens, G., Jaspers, J., Prangsma, M., \& Kanselaar, G. (2005). Coordination processes in computer supported collaborative writing. Computers in Human Behavior, 21, 463486.

Fischer, F., Bruhn, J., Gräsel, C., \& Mandl, H. (2002). Fostering collaborative knowledge construction with visualization tools. Learning and Instruction, 12, 213-232.

Frederiksen, J. R., \& White, B. Y. (2002). Conceptualizing and constructing linked models: Creating coherence in complex knowledge systems. In P. Brna, M. Baker, K. Stenning, \& A. Tiberghein (Eds.). In the role of communication in learning to model. (pp. 69-96). Mahwah, New Jersey: Lawrence Erlbaum Associates.

Gagné, R. M., Brigg, L. J., \& Wagner, W. W. (1992). Principles of instructional design. (4 ${ }^{\text {th }}$ ed.). Forth Worth: Harcourt Brace Jovanovich.

Hmelo-Silver, C. E., Duncan, R. G., \& Chinn, C. A. (2007). Scaffolding and achievement in problem-based and inquiry learning: A response to Kirschner, Sweller, and Clark (2006). Educational Psychologist, 42, 99-107.

Janssen, J. (2008). Using visualizations to support collaboration and coordination during computer-supported collaborative learning. Ph.D. thesis, Utrecht University, The Netherlands.

Jonassen, D. H. (2003). Using cognitive tools to represent problems. Journal of Research on Technology in Education, 35, 362-381.

Jonassen, D. H., \& Ionas, I. G. (2008). Designing effective support for causal reasoning. Educational Technology Research and Development, 56, 287-308.

Kenny, D. A., Kashy, D. A., \& Cook, W. L. (2006). Dyadic data analysis. New York/London: The Guilford Press. 
Kester, L., Kirschner, P. A., \& Corbalan, G. (2007). Designing support to facilitate learning in powerful electronic learning environments. Computers in Human Behavior, 23, 10471054.

Kirschner, F., Paas, F., \& Kirschner, P. A. (2009). Individual and group-based learning from complex cognitive tasks: Effects on retention and transfer efficiency. Computers in Human Behavior, 25, 306-314.

Kirschner, P. A., Beers, P. J., Boshuizen, H. P. A., \& Gijselaers, W. H. (2008). Coercing shared knowledge in collaborative learning environments. Computers in Human Behavior, 24, 403-420.

Kirschner, P. A., Martens, R. L., \& Strijbos, J. W. (2004). CSCL in higher education? A framework for designing multiple collaborative environments. In J. W. Strijbos, P. A. Kirschner, \& R. L. Martens (Eds.), What we know about CSCL, and implementing it in higher education (pp. 3-30). Boston: Kluwer Academic Publishers.

Kreijns, K., Kirschner, P. A., \& Jochems, W. (2003). Identifying the pitfalls for social interaction in computer-supported collaborative learning environments: A review of the research. Computers in Human Behavior, 19, 335-353.

Lazonder, A. W., \& Rouet, J. F. (2008). Information problem solving instruction: Some cognitive and metacognitive issues. Computers in Human Behavior, 24, 753-765.

Marjanovic, O. (2007). Using process-oriented, sequencing educational technologies: Some important pedagogical issues. Computers in Human Behavior, 23, 2742-2759.

Mercer, N., Littleton, K., \& Wegerif, R. (2004). Methods for studying the processes of interaction and collaborative activity in computer-based educational activities. Technology, Pedagogy and Education, 13, 195-212. 
Moos, D. C., \& Azevedo, R. (2008). Monitoring, planning, and self-efficacy during learning with hypermedia: The impact of conceptual scaffolds. Computers in Human Behavior, $24,1686-1706$.

Narciss, S., Proske, A., \& Koerndle, H. (2007). Promoting self-regulated learning in webbased learning environments. Computers in Human Behavior, 23, 1126-1144.

Ploetzner, R., Fehse, E., Kneser, C., \& Spada, H. (1999). Learning to relate qualitative and quantitative problem representations in a model-based setting for collaborative problem solving. Journal of the Learning Sciences, 8, 177-214.

Schnotz, W., \& Kürschner, C. (2008). External and internal representations in the acquisition and use of knowledge: visualization effects on mental model construction. Instructional Science, 36, 175-190.

Schiffrin, D. (1987). Discourse markers. Cambridge, MA: Cambridge University Press.

Slof, B., Erkens, E., Kirschner, P. A., \& Jaspers, J. G. M. (in press). Design and Effects of a Representational Scripting Tool on Group Performance. Educational Technology Research and Development.

Simon, H. L., Langley, P. W., \& Bradshaw, G. (1981). Scientific discovery as problem solving. Syntheses, 47, 1-27.

Spector, J. M. (2008). Cognition and learning in the digital age: Promising research and practice. Computers in Human Behavior, 24, 249-262.

Strijbos, J.-W., \& Fischer, F. (2007). Methodological challenges for collaborative learning research. Learning and Instruction, 17, 389-393.

Strijbos, J. W., Martens, R. L., Prins, F. J., \& Jochems, W. M. G. (2006). Content analysis: What are they talking about? Computers and Education, 46, 29-48.

Seufert, T. (2003). Supporting coherence formation in learning from multiple representations. Learning and Instruction, 13, 191-203. 
Suthers, D. D. (2006). Technology affordances for intersubjective meaning making: A research agenda for CSCL. International Journal of Computer-Supported Collaborative Learning, 1, 315-337.

Van Bruggen, J. M., Boshuizen, H. P. A., \& Kirschner, P. A. (2003). A cognitive framework for cooperative problem solving with argument visualization. In P. A. Kirschner, S. J. Buckingham-Shum, \& C. S. Carr (Eds.), Visualizing Argumentation: Software tools for collaborative and educational sense-making. (pp. 25-47). London: Springer.

Van der Linden, J. L., Erkens, G., Schmidt, H., \& Renshaw, P. (2000). Collaborative learning. In P. R. J. Simons, J. L. Van der Linden, \& T. Duffy (Eds.), New learning (pp. 1-19). Dordrecht: Kluwer Academic Publishers.

Van Drie, J. Van, Boxtel, C., Jaspers, J., \& Kanselaar, G. (2005). Effects of representational guidance on domain specific reasoning in CSCL. Computers in Human behavior, 21, $575-602$.

Van Merriënboer, J. J. G., Kester, L., \& Paas, F. (2006). Teaching complex rather than simple tasks: Balancing intrinsic and germane load to enhance transfer of learning. Applied Cognitive Psychology, 20, 343-352.

Veldhuis-Diermanse, A. E. (2002). CSCLearning? Participation, learning activities and knowledge construction in computer-supported collaborative learning in higher education. Unpublished Ph.D. thesis, Wageningen University, The Netherlands.

Vekiri, I. (2002). What is the value of graphical displays in learning? Educational Psychology Review, 14, 261-312.

Weinberger, A., Ertl, B., Fischer, F., \& Mandl, H. (2005). Epistemic and social scripts in computer-supported collaborative learning. Instructional Science, 33, 1-30. 

Table 1

Congruence between External Representation and Part-task Demands

\begin{tabular}{|c|c|c|c|}
\hline Problem phase & Task demand & ER & Representational guidance \\
\hline Problem orientation & $\begin{array}{l}\text { Determining core concepts and relating them to the } \\
\text { problem }\end{array}$ & $\begin{array}{l}\text { Conceptual } \\
\text { (static) }\end{array}$ & $\begin{array}{l}\text { Showing concepts and their } \\
\text { interrelationship }\end{array}$ \\
\hline Problem solution & Proposing multiple solutions to the problem & $\begin{array}{l}\text { Causal } \\
\text { (static) }\end{array}$ & $\begin{array}{l}\text { Showing causal relation between the } \\
\text { concepts and possible solutions }\end{array}$ \\
\hline Solution evaluation & $\begin{array}{l}\text { Determining suitability of the solutions and coming } \\
\text { to a final solution to the problem }\end{array}$ & $\begin{array}{l}\text { Simulation } \\
\text { (dynamic) }\end{array}$ & $\begin{array}{l}\text { Showing mathematical relation between } \\
\text { the concepts and enabling manipulation } \\
\text { of their value }\end{array}$ \\
\hline
\end{tabular}


Table 2

Overview of the Experimental Conditions

Problem phase Condition and provided ER

\begin{tabular}{lcccc}
\hline & Conceptual & Causal & Simulation & Match \\
& condition & condition & condition & Condition \\
\cline { 2 - 5 } & & & & \\
Problem orientation & Conceptual ER & Causal ER & Simulation ER & Conceptual ER \\
Problem solution & Conceptual ER & Causal ER & Simulation ER & Causal ER \\
Solution evaluation & Conceptual ER & Causal ER & Simulation ER & Simulation ER
\end{tabular}


Table 3

Coding and Category Kappa's $\left(K_{c}\right)$ of Students' Meta-cognitive, Cognitive and Off-task Activities

\begin{tabular}{|c|c|c|c|}
\hline Activities & Discourse topic & Discussion of & $K_{c}$ \\
\hline \multirow[t]{4}{*}{ Meta-cognitive } & & & .66 \\
\hline & Planning & the problem-solving strategy; how and when the group has to perform a specific activity & .50 \\
\hline & Monitoring & whether they have finished the part-tasks on time & .62 \\
\hline & Evaluating & the suitability of their problem-solving strategy & .66 \\
\hline \multirow[t]{4}{*}{ Cognitive } & & & .63 \\
\hline & Preparation & the goal of the problem-solving task and the different part-tasks & .64 \\
\hline & Executing & content-related topics and formulating / answers to the part-tasks & .70 \\
\hline & Ending & how, where, and when the answers to the part-tasks need to be registered & .53 \\
\hline \multirow[t]{3}{*}{ Off-task } & & & .73 \\
\hline & Social & non-task related topics & .74 \\
\hline & Technical & problems with the CSCL-environment & .70 \\
\hline
\end{tabular}


Table 4

Coding and Category Kappa's (Kc) MEPA-filter of Students' Discussion of Concepts, Solutions and Relations

\begin{tabular}{|c|c|c|c|}
\hline Categories & Subcategories & Discussion of & $K_{c}$ \\
\hline \multirow[t]{6}{*}{ Concepts } & & & .86 \\
\hline & Sales & how many products are sold/have to produced & .87 \\
\hline & Selling price & what it costs to produce and sell a product and what the customer has to pay for the product & .50 \\
\hline & Costs & what the overall costs of the company are & .83 \\
\hline & Turnover & what the total income of the company is & .93 \\
\hline & Company result & whether it is profitable to run the company & .91 \\
\hline \multirow[t]{4}{*}{ Solutions } & & & .86 \\
\hline & Changing costs & how the overall costs can be decreased & .86 \\
\hline & Changing turnover & how the turnover can be increased & .90 \\
\hline & Changing both & the combining of the other two solutions & .80 \\
\hline \multirow[t]{4}{*}{ Relations } & & & .70 \\
\hline & Conceptual & the definition /meaning of a concept/solution & .73 \\
\hline & Causal & the causal relationship within /between concepts/solutions & .83 \\
\hline & Mathematical & the quantitative relationships within/between concepts & .73 \\
\hline
\end{tabular}


Table 5

Coding of Students' Communicative Activities

\begin{tabular}{|c|c|c|c|}
\hline Activities & Dialogue Act & Description & Example discourse marker \\
\hline \multirow[t]{5}{*}{ Focusing } & Elicitative proposal for action & Proposition for action & Let's start with the first part-task? \\
\hline & Elicitative question open & $\begin{array}{l}\text { Open question with a lot of } \\
\text { alternatives }\end{array}$ & $\begin{array}{l}\text { Shall we fist look at the description of the assignment or at } \\
\text { the description of the part-tasks? }\end{array}$ \\
\hline & Imperative action & Command to perform an action & Finish the answer to the second part-task \\
\hline & Imperative focus & Command for attention & Look at the representational tool! \\
\hline & Elicitative question verify & $\begin{array}{l}\text { Question that can only be } \\
\text { answered with yes or no }\end{array}$ & Do you refer to the company result?? \\
\hline \multirow[t]{4}{*}{ Checking } & Elicitative question set & $\begin{array}{l}\text { Question where the alternatives } \\
\text { are already given (set) }\end{array}$ & Are you for or against increasing sales? \\
\hline & Responsive confirm & Confirming answer & Yes, we indeed should start a PR-campaign \\
\hline & Responsive deny & Denying answer & No, that is not a good solution \\
\hline & Responsive accept & Accepting answer & Oh, Yes that OK \\
\hline \multirow[t]{5}{*}{ Argumentation } & Argumentative reason & Reason & Because this solution does not affect our costs \\
\hline & Argumentative against & Objection & But this would cost more money \\
\hline & Argumentative conditional & Condition & If we increase the selling price... \\
\hline & Argumentative then & Consequence & Then the cost price decreases \\
\hline & Argumentative disjunctive & Disjunctive & We can increase the actual sales through a PR-campaign or \\
\hline
\end{tabular}


by decreasing the selling price or by ....

Argumentative conclusion Conclusion Thus we can conclude that the third solution leads to the best company result. 
Table 6

Items and Reliability of Group Learning-task Performance

\begin{tabular}{|c|c|c|c|}
\hline Criteria & Description & Items & $\alpha$ \\
\hline Suitability & Whether the groups' answers were suited to the different part-tasks & 9 & .61 \\
\hline Elaboration & $\begin{array}{l}\text { Number of different business-economics concepts or financial consequences incorporated in the answers } \\
\text { to the different part-tasks }\end{array}$ & 9 & .53 \\
\hline Justification & Whether the groups justified their answers to the different part-tasks & 9 & .73 \\
\hline Correctness & $\begin{array}{l}\text { Whether the groups used the business-economics concepts and their interrelationships correctly in their } \\
\text { answers to the different part-tasks }\end{array}$ & 9 & .68 \\
\hline Continuity & Whether the groups made proper use of the answers from a prior problem phase & 2 & .67 \\
\hline Quality advice & $\begin{array}{l}\text { Whether the groups gave a proper final advice } \\
\text { - Number of business-economics concepts incorporated in the advice } \\
\text { - Number of financial consequences incorporated in the advice } \\
\text { - Whether the final answer conformed to the guidelines provided }\end{array}$ & 3 & .71 \\
\hline Total score & Overall score on the complex learning-task performance & 41 & .89 \\
\hline
\end{tabular}


Table 7

Multilevel Analyses for Effects of Match Condition versus Non-matched Conditions concerning Students' Metacognitive, Cognitive and Off-task activities

\begin{tabular}{|c|c|c|c|c|c|c|c|}
\hline & $\begin{array}{l}\text { Conceptual } \\
\text { condition } \\
\left(n_{\text {student }}=22\right)\end{array}$ & $\begin{array}{l}\text { Causal } \\
\text { condition } \\
\left(n_{\text {student }}=24\right)\end{array}$ & $\begin{array}{l}\text { Simulation } \\
\text { condition } \\
\left(n_{\text {student }}=23\right)\end{array}$ & $\begin{array}{l}\text { Match } \\
\text { condition } \\
\left(n_{\text {student }}=24\right)\end{array}$ & $\begin{array}{l}\text { Effects } 1 \\
\text { conditio } \\
\left(N_{\text {student }}=\right.\end{array}$ & & \\
\hline & $M(S D)$ & $M(S D)$ & $M(S D)$ & $M(S D)$ & $\chi^{2}(3)$ & $\beta$ & $S E$ \\
\hline Meta-cognitive & $18.96(8.72)$ & $20.33(14.06)$ & $21.95(26.39)$ & $32.61(33.76)$ & 17.08 & 6.82 & 4.84 \\
\hline Planning & $2.59(1.59)$ & $1.62(1.32)$ & 1.45 (1.97) & $2.82(2.87)$ & 4.32 & 0.11 & 0.32 \\
\hline Monitoring & $12.75(6.41)$ & $15.23(12.00)$ & $16.23(20.35)$ & $24.75(28.21)$ & 16.00 & 6.01 & 3.85 \\
\hline Evaluating & $3.62(2.28)$ & $3.48(2.52)$ & $4.27(5.82)$ & $5.04(5.79)$ & 5.22 & 0.71 & 0.89 \\
\hline Cognitive & $16.00(17.44)$ & $10.19(7.03)$ & $7.41(6.93)-$ & $18.32(13.60)+$ & 116.56 & 1.04 & 2.32 \\
\hline Preparation & $2.92(3.64)$ & $1.29(1.49)$ & $1.50(1.87)$ & $2.25(2.35)$ & 4.10 & 0.35 & 0.41 \\
\hline Executing & $10.67(16.23)$ & $7.00(6.30)$ & $5.00(4.77)-$ & $12.64(9.84)+$ & 13.58 & 0.92 & 1.89 \\
\hline Ending & $2.41(2.06)$ & $1.90(2.02)$ & $0.91(1.27)-$ & $3.43(4.17)+$ & 7.46 & 0.49 & 0.47 \\
\hline Off-task & $7.29(5.41)$ & $4.95(4.33)$ & $5.73(5.51)$ & $8.46(6.24)$ & 8.69 & 0.61 & 1.12 \\
\hline Social & $5.62(4.41)$ & $4.05(4.05)$ & $4.77(4.84)$ & $5.79(4.53)$ & 5.54 & 0.10 & 0.90 \\
\hline Technical & $1.67(1.61)$ & $0.90(0.94)-$ & $0.95(1.17)-$ & $2.68(2.16)+$ & 8.00 & $0.50^{*}$ & 0.31 \\
\hline
\end{tabular}

Notes. ${ }^{*} p<.05$; if match condition significantly $>$ a mismatch condition than the match condition is indicated with a 
+ and the mismatch condition with a - 
Table 8

Multilevel Analyses for Effects of Match Condition versus Non-matched Conditions concerning Students'

Discussion of Concepts, Solutions and Relations

\begin{tabular}{|c|c|c|c|c|c|c|c|}
\hline & $\begin{array}{l}\text { Conceptual } \\
\text { condition } \\
\left(n_{\text {student }}=22\right)\end{array}$ & $\begin{array}{l}\text { Causal } \\
\text { condition } \\
\left(n_{\text {student }}=24\right)\end{array}$ & $\begin{array}{l}\text { Simulation } \\
\text { condition } \\
\left(n_{\text {student }}=23\right)\end{array}$ & $\begin{array}{l}\text { Match } \\
\text { condition } \\
\left(n_{\text {student }}=23\right)\end{array}$ & $\begin{array}{l}\text { Effects } \\
\text { conditi } \\
\text { ( } N_{\text {studen }}\end{array}$ & $\begin{array}{l}\text { match } \\
\text { ion } \\
=92)\end{array}$ & \\
\hline & $M(S D)$ & $M(S D)$ & $M(S D)$ & $M(S D)$ & $\chi^{2}(3)$ & $\beta$ & $S E$ \\
\hline Concepts & $21.59(22.30)$ & $18.58(18.73)-$ & $19.86(19.91)-$ & $32.25(20.81)+$ & 16.81 & 5.27 & 3.82 \\
\hline Sales & $3.93(6.64)-$ & $2.29(2.49)-$ & $5.32(5.62)$ & $7.86(7.00)+$ & 12.49 & $1.97^{*}$ & 1.00 \\
\hline Selling price & $1.07(1.66)$ & $1.21(1.89)$ & $1.18(2.47)$ & $2.07(2.54)$ & 1.40 & 0.51 & 0.37 \\
\hline Costs & $5.70(7.75)-$ & $6.17(7.40)$ & $5.00(6.03)-$ & $10.57(9.29)+$ & 11.72 & $2.46^{*}$ & 1.44 \\
\hline Turnover & $3.78(3.47)$ & $3.17(3.24)$ & $2.54(3.00)$ & $4.39(3.21)$ & 4.36 & 0.29 & 0.56 \\
\hline Company result & $7.11(8.73)$ & $5.75(7.02)$ & $5.82(6.34)$ & $7.36(4.83)$ & 6.55 & 0.05 & 1.20 \\
\hline Solutions & $14.19(14.60)$ & $15.12(17.00)$ & $14.00(14.26)$ & $24.11(19.81)$ & 15.46 & $4.96^{*}$ & 2.95 \\
\hline Changing costs & $5.52(8.56)-$ & $5.13(9.03)-$ & $7.43(9.85)$ & $12.00(12.01)+$ & 12.59 & $3.18^{*}$ & 1.92 \\
\hline Changing turnover & $6.11(6.29)$ & $6.71(6.62)$ & $4.86(4.62)-$ & $9.54(9.11)+$ & 10.40 & 1.74 & 1.13 \\
\hline Changing both & $2.56(3.23)$ & $3.29(6.52)$ & $1.71(1.98)$ & $2.57(2.99)$ & 3.57 & 0.02 & 0.64 \\
\hline Relations & $25.89(20.65)$ & $23.17(19.23)$ & $26.36(21.63)$ & $36.63(22.35)$ & 16.37 & 5.32 & 3.85 \\
\hline Conceptual & $6.48(4.76)$ & $4.38(4.17)$ & $7.43(5.77)$ & $8.96(6.08)$ & 10.38 & 1.26 & 0.93 \\
\hline
\end{tabular}




\begin{tabular}{lrrrrrrr} 
Causal & $16.74(14.97)$ & $14.46(13.94)$ & $15.57(13.53)$ & $21.86(14.18)$ & 12.87 & 2.54 & 2.52 \\
Mathematical & $2.67(3.13)-$ & $4.33(4.82)$ & $3.36(3.76)-$ & $5.82(5.20)+$ & 7.95 & $1.52^{*}$ & 0.72 \\
\hline
\end{tabular}

Notes. ${ }^{*} p<.05$; if match condition significantly $>$ a mismatch condition than the match condition is indicated with $\mathrm{a}+$ and the mismatch condition with a - 
Table 9

Multilevel Analyses for Effects of Match Condition versus Non-matched Conditions concerning Students'

Communicative Activities

\begin{tabular}{|c|c|c|c|c|c|c|}
\hline & Conceptual & Causal & Simulation & Match & Effects match & \\
\hline & condition & condition & condition & condition & condition & \\
\hline & $\left(n_{\text {student }}=22\right)$ & $\left(n_{\text {student }}=24\right)$ & $\left(n_{\text {student }}=23\right)$ & $\left(n_{\text {student }}=23\right)$ & $\left(N_{\text {student }}=92\right)$ & \\
\hline & $M(S D)$ & $M(S D)$ & $M(S D)$ & $M(S D)$ & $\chi^{2}(3)$ & $S E$ \\
\hline Coordination & $109.92(56.60)$ & $101.82(54.65)$ & $145.15(88.45)$ & $170.32(137.58)$ & 26.3327 .09 & 17.96 \\
\hline Focusing & $21.75(14.00)$ & $20.06(10.63)$ & $28.55(16.46)$ & $32.73(27.11)$ & $15.93 \quad 4.63$ & 3.49 \\
\hline Checking & $55.04(26.06)$ & $51.41(27.32)$ & $69.60(44.00)$ & $83.00(66.48)$ & $21.71 \quad 12.83$ & 8.80 \\
\hline Argumentation & $33.12(24.06)-$ & $30.35(21.32)-$ & $47.00(32.10)$ & $54.59(49.28)+$ & $20.5210 .26^{*}$ & 6.20 \\
\hline
\end{tabular}

Notes. ${ }^{*} p<.05$; if match condition significantly $>$ a mismatch condition than the match condition is indicated with a +

and the mismatch condition with a - 
Table 10

One-way Multivariate Analysis of Variance for Effects of Match Condition versus

Non-matched Conditions concerning Group Learning-task Performance

\begin{tabular}{lcccc} 
Criteria & $\begin{array}{c}\text { Conceptual } \\
\text { condition } \\
\left(n_{\text {group }}=8\right)\end{array}$ & $\begin{array}{c}\text { Causal } \\
\text { condition } \\
\left(n_{\text {group }}=8\right)\end{array}$ & $\begin{array}{c}\text { Simulation } \\
\text { condition } \\
\left(n_{\text {group }}=8\right)\end{array}$ & $\begin{array}{c}\text { Match } \\
\text { condition } \\
\left(n_{\text {group }}=8\right)\end{array}$ \\
\cline { 2 - 5 } & $M(S D)$ & $M(S D)$ & $M(S D)$ & $M(S D)$ \\
\cline { 2 - 5 } Suitability $^{*}$ & $12.25(2.49)-$ & $15.12(1.64)$ & $13.88(3.36)-$ & $15.75(2.42)+$ \\
Elaboration $^{*}$ & $6.38(3.74)$ & $8.89(2.70)$ & $6.37(2.83)$ & $8.38(2.33)$ \\
Justification $^{*}$ & $3.50(1.69)-$ & $6.88(3.56)$ & $4.12(2.70)-$ & $7.50(2.62)+$ \\
Correctness $^{*}$ & $5.50(2.45)-$ & $8.25(3.69)$ & $7.12(1.96)-$ & $9.25(2.05)+$ \\
Continuity & $2.50(1.41)$ & $3.12(1.13)$ & $3.00(1.31)$ & $3.62(0.52)$ \\
Quality advice $^{*}$ & $2.75(1.04)$ & $4.88(1.64)$ & $5.12(2.48)$ & $4.25(1.28)$ \\
Total score $^{*}$ & $32.88(10.40)-$ & $47.13(12.30)$ & $39.62(0.39)-$ & $48.75(7.27)+$
\end{tabular}

Notes. ${ }^{*} p<.05$; if match condition significantly $>$ a mismatch condition than the

match condition is indicated with a + and the mismatch condition with a - 


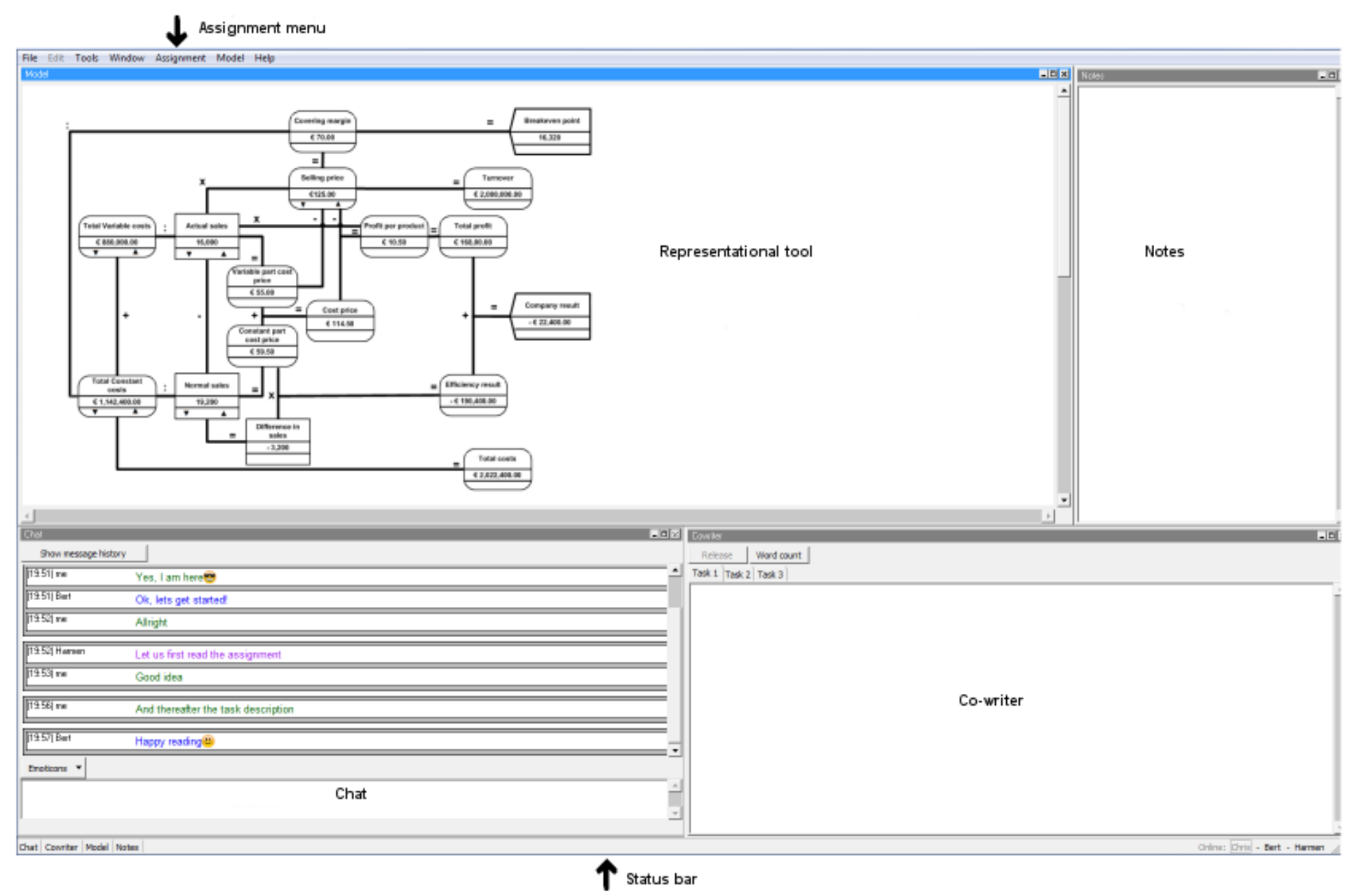

Fig 1. Screenshot of the VCRI-environment (simulation tool) 


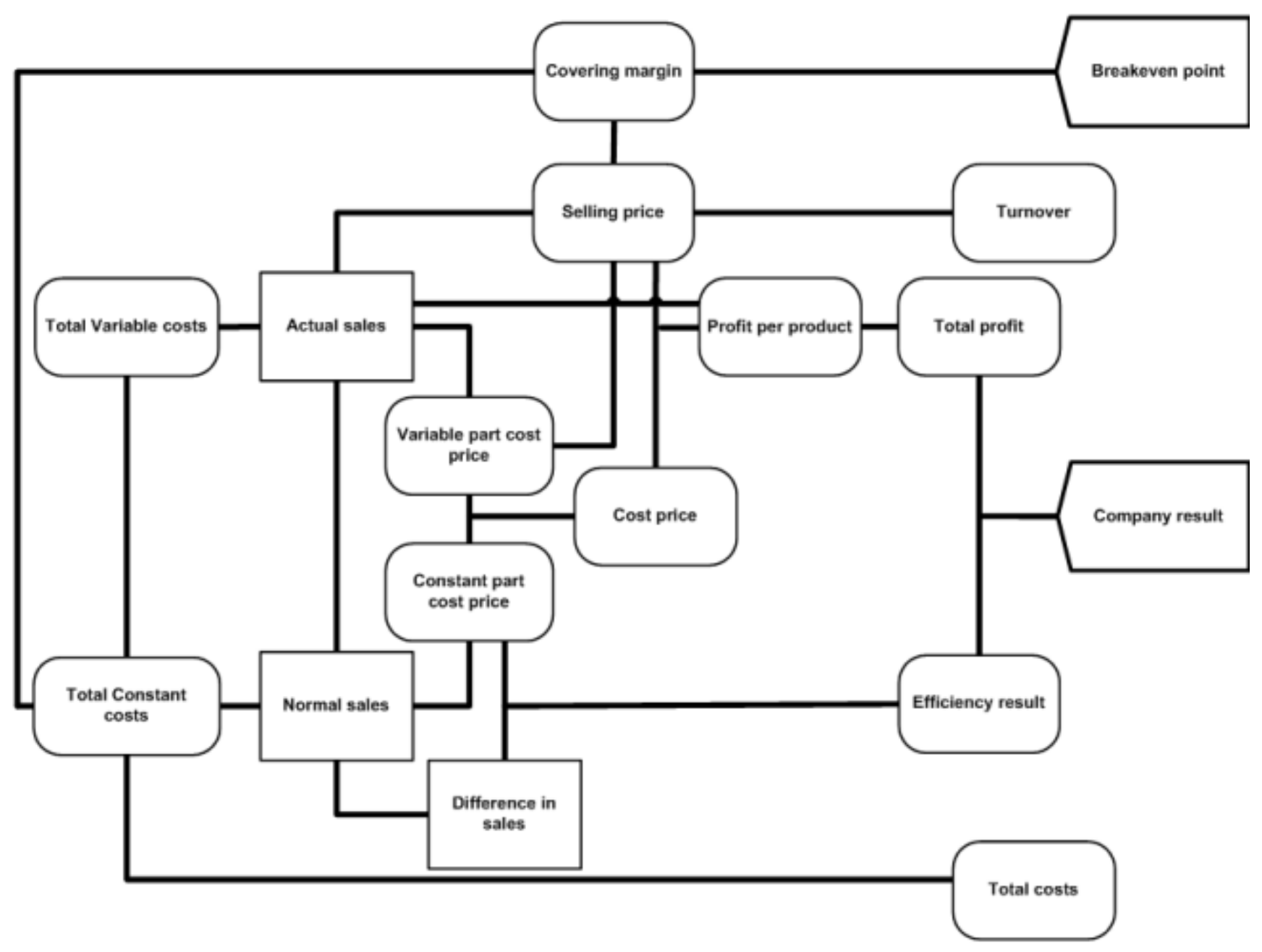

Fig 2. Conceptual representation (expert model) 


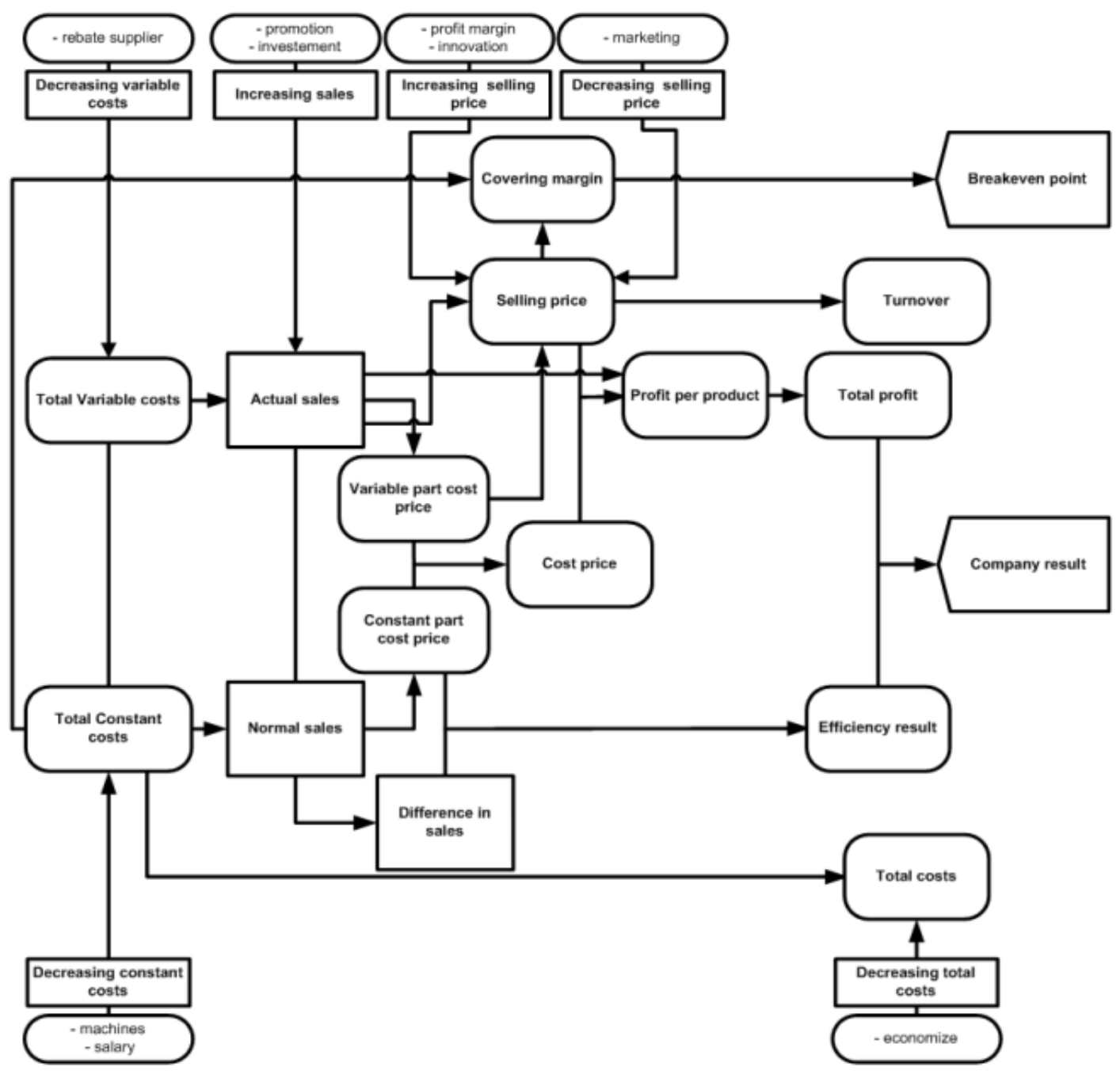

Fig 3. Causal representation (expert model) 


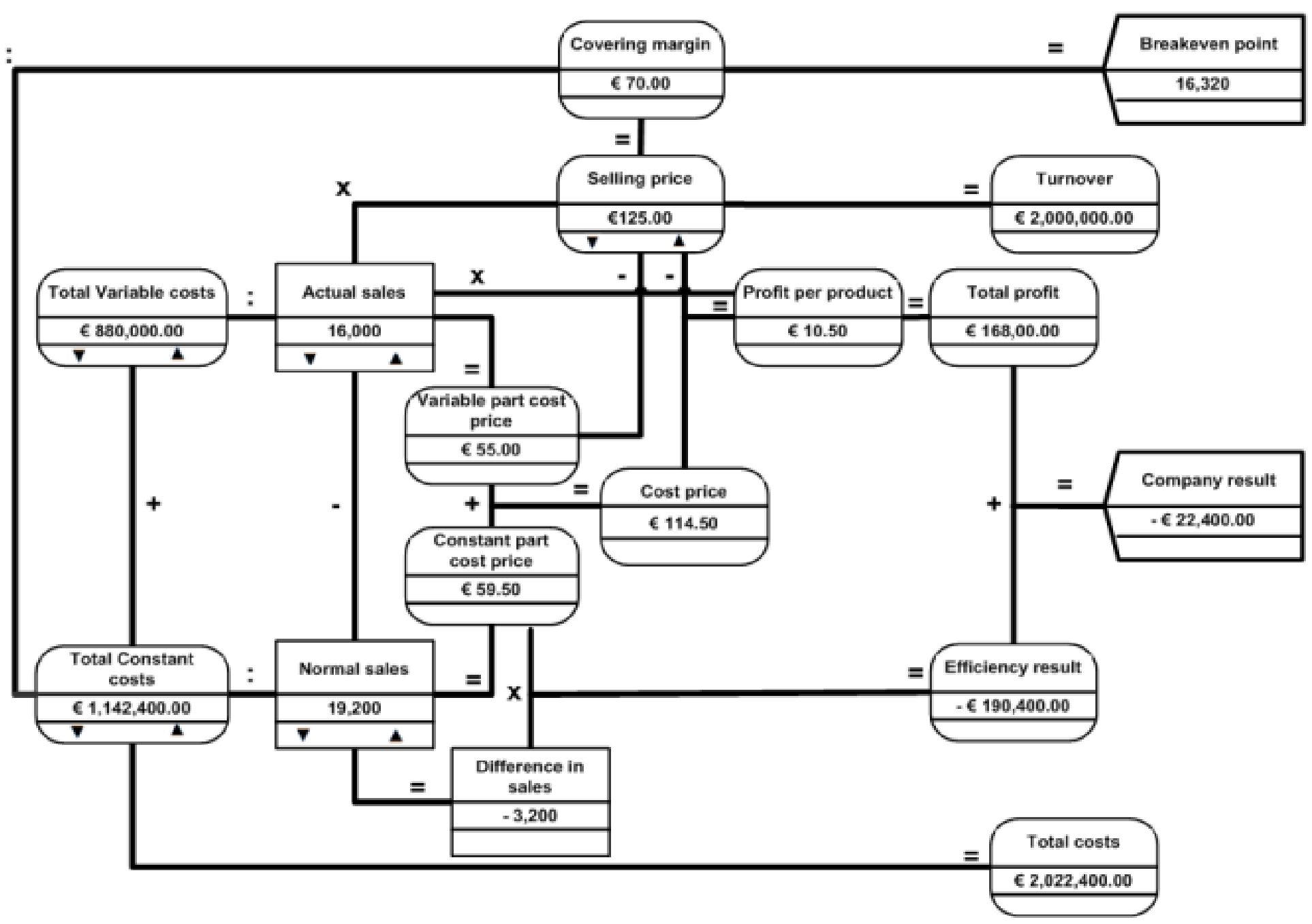

Fig 4. Simulation representation (expert model) 